\title{
Closing the diarrhoea diagnostic gap in Indian children by the application of molecular techniques
}

\begin{abstract}
Correspondence
G. Kang

gkang@cmcvellore.ac.in
\end{abstract}

Received 8 May 2008

Accepted 16 July 2008

\author{
S. S. R. Ajjampur, ${ }^{1}$ P. Rajendran, ${ }^{1}$ S. Ramani, ${ }^{1}$ I. Banerjee, ${ }^{1}$ B. Monica,${ }^{1}$ \\ P. Sankaran, ${ }^{1}$ V. Rosario, ${ }^{1}$ R. Arumugam, ${ }^{1}$ R. Sarkar, ${ }^{1}$ H. Ward ${ }^{2}$ \\ and G. Kang ${ }^{1}$
}
${ }^{1}$ Department of Gastrointestinal Sciences, Christian Medical College, Vellore 632004, India
${ }^{2}$ Division of Geographic Medicine and Infectious Diseases, Tufts-New England Medical Center, Boston, MA 02421, USA

\begin{abstract}
A large proportion of diarrhoeal illnesses in children in developing countries are ascribed to an unknown aetiology because the only available methods, such as microscopy and culture, have low sensitivity. This study was aimed at decreasing the diagnostic gap in diarrhoeal disease by the application of molecular techniques. Faecal samples from 158 children with and 99 children without diarrhoea in a hospital in South India were tested for enteric pathogens using conventional diagnostic methods (culture, microscopy and enzyme immunoassays) and molecular methods (six PCR-based assays). The additional use of molecular techniques increased identification to at least one aetiological agent in $76.5 \%$ of diarrhoeal specimens, compared with $40.5 \%$ using conventional methods. Rotavirus (43.3\%), enteropathogenic Escherichia coli (15.8\%), norovirus (15.8\%) and Cryptosporidium spp. (15.2\%) are currently the most common causes of diarrhoea in hospitalized children in Vellore, in contrast to a study conducted two decades earlier in the same hospital, where bacterial pathogens such as Shigella spp., Campylobacter spp. and enterotoxigenic E. coli were more prevalent. Molecular techniques significantly increased the detection rates of pathogens in children with diarrhoea, but a more intensive study, testing for a wider range of infectious agents and including more information on non-infectious causes of diarrhoea, is required to close the diagnostic gap in diarrhoeal disease.
\end{abstract}

\section{INTRODUCTION}

Diarrhoea is the cause of death in almost $23 \%$ of Indian children who die before the age of 5 (Jones et al., 2006). It is one of the most common childhood illnesses, with every child under 5 years of age in the developing world experiencing around three episodes of diarrhoea per year (Kosek et al., 2003). In addition to the mortality and morbidity associated with diarrhoea as a dehydrating illness, chronic and recurrent diarrhoea in early childhood has been shown to have long-term effects such as stunting and developmental delays caused by poor nutrient absorption (Berkman et al., 2002). Although more than 200 viral, bacterial and parasitic causes of diarrhoea have been identified to date, only a handful of aetiological agents cause the vast majority of diarrhoeal diseases in children in the developing world and include rotavirus, diarrhoeagenic Escherichia coli, Campylobacter jejuni, Shigella spp., nontyphoidal Salmonella, Giardia lamblia, Cryptosporidium spp.

Abbreviations: DAEC, diffusely adherent Escherichia coli; EAEC, enteroaggregative E. coli; EHEC, enterohaemorrhagic E. coli; EIEC, enteroinvasive E. coli; EPEC, enteropathogenic E. coli; ETEC, enterotoxigenic E. coli. and Entamoeba histolytica (O'Ryan et al., 2005). In earlier studies in developing countries, astrovirus, enteric adenovirus and calicivirus were also seen, but these had a lesser relative contribution due to higher rates of bacterial and parasitic infections (O'Ryan et al., 2005). Identifying the enteric pathogens that contribute significantly to the causes of diarrhoea in children will help to provide an accurate and region-specific estimate of disease burden. Traditional methods to detect the aetiological agents of diarrhoea have ranged from microscopy and bacterial culture to immunoassays, and vary for each enteric pathogen. This has also resulted in a large proportion of samples being ascribed to an unknown aetiology. In contrast, PCR-based methods provide an easily performed, rapid and uniform method of testing for a wide range of pathogens. In this study, we aimed to use six sensitive PCR assays for common enteric pathogens to increase the detection of aetiological agents of diarrhoea in children in Vellore, India, thereby narrowing the diagnostic gap. Faecal samples from children with diarrhoea and from children presenting to hospital for reasons other than those related to the gastrointestinal tract were tested for a wide range of enteric pathogens using conventional techniques and six PCR assays. 


\section{METHODS}

Study subjects. In this study, 158 children aged under 5 years admitted to hospital with diarrhoea between January and December 2003 were prospectively recruited into the study after obtaining informed consent from their parent/guardian. Diarrhoea was defined as the passage of three or more watery stools in a $24 \mathrm{~h}$ period. A minimum of two diarrhoea-free days was considered to delineate between two episodes of diarrhoea (Monica et al., 2007). The severity of diarrhoea was assessed using the 20-point scale of the Vesikari scoring system (Ruuska \& Vesikari, 1990), which has been used in previous studies on hospitalized children (Kurugol et al., 2003; Kang et al., 2004). Ninety-nine children aged 5 years or under admitted to the hospital for causes other than diarrhoea between July and September 2004 were included as controls. Stool samples were collected from the study subjects and transported immediately to the laboratory. The study was approved by the institutional review board.

Diagnostic methods. All stool samples were screened for stool parasites by light microscopy and for Cryptosporidium spp. by a modified acid-fast staining (Henriksen \& Pohlenz, 1981). Stool culture was carried out to identify the following bacterial pathogens: Salmonella spp., Shigella spp., Vibrio cholerae and enteropathogenic E. coli (EPEC). Samples were cultured on blood agar and selective media including sorbitol MacConkey agar, xylose lysine deoxycholate agar and thiosulphate citrate bile salts sucrose (TCBS) agar and incubated at $37^{\circ} \mathrm{C}$ overnight. Samples were also inoculated in selenite $\mathrm{F}$ broth and alkaline peptone water for 18 and $4 \mathrm{~h}$, respectively, for amplification of Salmonella and Vibrio spp. and subcultured on MacConkey agar and TCBS agar, respectively. For identification of EPEC, slide agglutination with antisera to common EPEC $\mathrm{O}$ antigens was carried out on eight to ten smooth, grey colonies on blood agar and lactose-fermenting colonies on MacConkey agar. All samples were also screened by ELISA for group A rotavirus (Rotavirus IDEIA; Bio-Rad). In addition, samples from children with diarrhoea were also screened for adenovirus and astrovirus (Ridascreen; RBiopharm) and for enterohaemorrhagic E. coli (EHEC) verotoxin by ELISA (Ridascreen; R-Biopharm).

Molecular methods. Nucleic acid was extracted from faecal samples using the method of Boom et al. (1990) for RNA and using a QIAamp DNA Stool Mini kit for DNA (Qiagen). The RNA extracts were reverse transcribed and the cDNA and DNA were both stored at $-20{ }^{\circ} \mathrm{C}$ until further testing. PCRs were then carried out for cases and controls for the following pathogens, using previously published primers and protocols (Table 1): rotavirus, norovirus (genogroups 1 and 2), Campylobacter spp. (coli and jejuni), Cryptosporidium spp. and diarrhoeagenic E. coli, which included EPEC, EHEC, enteroinvasive E. coli (EIEC), diffusely adherent E. coli (DAEC), enteroaggregative E. coli (EAEC) and enterotoxigenic E. coli (ETEC).

Statistical analysis. All laboratory data were entered in an Excel spreadsheet and analysis was carried out using SPSS 12.0 for Windows. Analysis was primarily descriptive and $\chi^{2}$ tests were used to compare the conventional and molecular methods for detection of pathogens. For analysis of the severity of diarrhoea, Vesikari scores were classified as mild, moderate or severe (Ruuska \& Vesikari, 1990), and the number of children with mild and moderate diarrhoea was compared with the number of children with severe diarrhoea using Fisher's exact test.

\section{RESULTS AND DISCUSSION}

Among the 158 children with diarrhoea, $76.5 \%$ (121/158) had at least one aetiological agent when both conventional and molecular methods were employed, compared with only $40.5 \%(64 / 158)$ when only conventional methods were used. Rotavirus ( $43.3 \%$ ) was the most common cause of diarrhoea, followed by EPEC (15.8\%), norovirus (15.8\%) and Cryptosporidium spp. (15.2\%) when results from both conventional methods and molecular methods were collated. The distribution of the other aetiological agents identified in children with and without diarrhoea is listed in Table 2. A larger proportion of children with diarrhoea had at least one viral $(53.2 \%, 84 / 158)$ aetiological agent identified in their stool sample, when compared with bacterial $(30.4 \%, 48 / 158)$ or parasitic $(17.1 \%, 27 /$ 158) agents.

When conventional and molecular methods were compared for the detection of individual pathogens such as rotavirus and Cryptosporidium spp. among the children

Table 1. PCR amplification targets for enteric pathogens

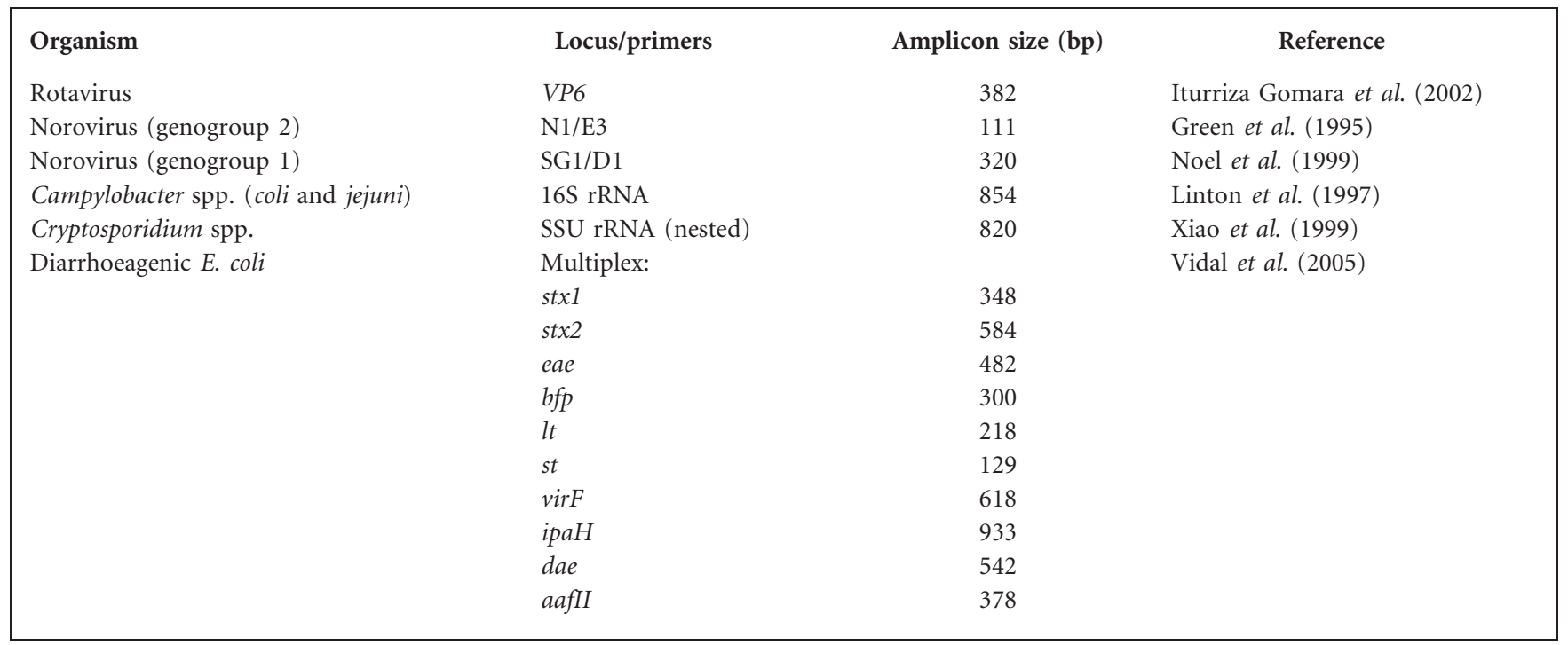


Table 2. Distribution of enteric pathogens in children with and without diarrhoea

\begin{tabular}{|lcc|}
\hline Organism & $\begin{array}{c}\text { No. of cases } \\
(\boldsymbol{n}=\mathbf{1 5 8})\end{array}$ & $\begin{array}{c}\text { No. of controls } \\
(\boldsymbol{n}=\mathbf{9 9})\end{array}$ \\
\hline Viral agents & & \\
Rotavirus ELISA & $40(25.3 \%)$ & $4(4 \%)$ \\
Rotavirus PCR & $58(36.9 \%)$ & $6(6.9 \%)$ \\
Norovirus PCR & $25(15.8 \%)$ & $7(7 \%)$ \\
Astrovirus ELISA $\dagger$ & $3(1.9 \%)$ & $\mathrm{ND}$ \\
Adenovirus ELISA $\dagger$ & $10(6.4 \%)$ & $\mathrm{ND}$ \\
Bacterial agents & & $3(3.1 \%)$ \\
Campylobacter PCR & $9(5.7 \%)$ & $\mathrm{ND}$ \\
EHEC ELISA & $4(2.5 \%)$ & $2(11.4 \%)$ \\
Multiplex E. coli PCR & $45(28.0 \%)$ & $2(2 \%)$ \\
EHEC & $3(1.9 \%)$ & $5(5 \%)$ \\
EPEC & $25(15.8 \%)$ & $1(1 \%)$ \\
ETEC & $10(6.3 \%)$ & $11 \%)$ \\
DAEC & $2(1.2 \%)$ & 0 \\
EIEC & $3(1.8 \%)$ & $2(2.04 \%)$ \\
EAEC & $2(1.2 \%)$ & 0 \\
Stool culture & & 0 \\
$\quad$ Vibrio cholerae & $1(0.6 \%)$ & \\
Shigella spp. & $1(0.6 \%)$ & \\
Protozoan agents & & \\
Microscopy & $7(4.4 \%)$ & \\
$\quad$ Cryptosporidium spp. & $1(0.63 \%)$ & \\
Giardia lamblia & $21(13.3 \%)$ & \\
Cryptosporidium PCR & & \\
\hline
\end{tabular}

ND, Not done.

${ }^{\star}$ Rotavirus PCR was carried out on samples from 157 cases and 87 controls.

$\dagger$ Astrovirus and adenovirus ELISA was carried out on 156 cases.

with diarrhoea, it was found that the detection rates were significantly higher when molecular methods were applied ( $43.3 \%$ and $15.2 \%$, respectively) compared with the use of conventional methods alone $(25.3 \%$ and $4.4 \%$, respectively) $(P<0.001)$. The increase in detected cases of rotaviral diarrhoea due to the increased sensitivity of PCR over ELISA has been demonstrated previously (Husain et al., 1995; Buesa et al., 1996). The limit of detection of rotavirus by ELISA, defined as $7.8 \times 10^{5}$ viral particles $(\mathrm{ml} \mathrm{stool})^{-1}$ by the manufacturer, is also lower than that of PCR, which has been determined to be in the range of $10^{4}-10^{5}$ p.f.u. $\mathrm{ml}^{-1}$ (DiStefano et al., 2005). However, among the 40 rotavirus ELISA-positive cases, nine were PCR-negative. This could be attributed to false positivity by ELISA (Kang et al., 2004) or to degradation of viral RNA in stool samples during storage. The ELISA was carried out on fresh stool samples, whereas the extraction method of Boom et al. (1990) and PCR were carried out in batches on stored samples. In EPEC-positive cases, none of the samples were positive by the conventional method of $\mathrm{O}$-antigen typing of coliform colonies from stool culture, but a large number $(25 / 158,15.8 \%)$ were detected by PCR for the eae gene, of which 18 were also positive by PCR for the $b f p$ gene. However, for EHEC, $2.5 \%$ of cases were positive by ELISA, with only a $1.9 \%$ increment when PCR was applied, with no overlap of cases detected by each method. The poor sensitivity of the multiplex PCR for EHEC could possibly also have been due to deterioration of DNA during storage, although this was not seen for EPEC. The time of testing and storage should be taken into account when planning future studies where a combination of conventional culture or ELISA and molecular techniques, such as PCR, may be required to identify aetiological agents, as the sensitivity of these methods depends on rapidity of testing and appropriate storage.

The combination of molecular and conventional methods considerably increased the number of mixed infections (two or more pathogens) identified in diarrhoeal samples to $31.6 \%(50 / 158)$. Of the 158 children, 61 were found to be infected with one pathogen and only three were identified as being infected with two pathogens when conventional methods alone were used. When molecular techniques alone were considered, 69 children had one pathogen identified, 30 had two pathogens identified and 10 had three or more pathogens identified. However, among the children identified as having only one pathogen, 12 samples were positive by a conventional ELISA test only (two EHEC, five adenoviral and five rotaviral infections). Among the children with more than one aetiological agent, 25 were infected with a combination of viral and bacterial agents, five with bacterial and parasitic agents, and nine with viral and parasitic agents. The most frequently occurring co-infections seen among children with diarrhoea were due to a combination of rotavirus together with norovirus $(8.9 \%)$, EPEC (4.4\%), ETEC (3.7\%) or Campylobacter spp. (3.2\%). High rates of co-infection with EPEC and ETEC in rotaviral diarrhoea have also been reported by other workers.

Among the rotavirus-positive cases, the number of children with mild and moderate or severe diarrhoea was compared in cases that were PCR-positive but ELISA-negative and cases that were both ELISA- and PCR-positive using the Vesikari scores and there was no significant difference (Fisher's exact test, $P=0.525$ ). Scores were available for 45 of the 58 children (30/58 were both ELISA- and PCRpositive, and 28 were PCR-positive but ELISA-negative). A similar comparison of nine ELISA-positive but PCRnegative cases with the 30 cases for whom both ELISA and PCR were positive also showed no significant difference $(P=0.52$, Fisher's exact test; data for $31 / 39$ ELISA-positive). Other workers have, however, identified differences in severity of diarrhoea based on ELISA or PCR positivity (Pang et al., 1999). In this study, there was also no significant difference in the number of children with mild, moderate or severe diarrhoea when children infected with a single pathogen were compared with those with multiple pathogens $(P=0.075)$.

The study demonstrated a $27.7 \%$ occurrence of asymptomatic enteric infections in children without diarrhoea 
when the results of both conventional and molecular tests were combined. The most common asymptomatic infections detected among the children without diarrhoea were due to rotavirus (10.1\%), norovirus (7\%) and ETEC (5.2\%). Only five children had more than one pathogen detected. Among the 99 controls, 87 samples were available for rotavirus PCR and six were found to be positive. Among the 87 samples tested, two were ELISA-positive but PCR-negative. The application of PCR resulted in only a slight increase in the numbers of asymptomatic rotaviral infections detected (from four by ELISA to six by PCR) compared with cases of symptomatic infections (from 40 by ELISA to 58 by PCR), supporting the higher sensitivity of PCR, but also indicating that the increased rates of detection by PCR are not solely due to identification of rotavirus infection not associated with disease.

Levine (2006) stated that the comparison of isolation rates of specific agents from cases versus controls allows one to calculate a 'pathogenicity index' (Table 3). Comparison of prevalence rates in children with and without diarrhoea for pathogens that had been tested for in both groups allowed an estimation of pathogenicity indices in this study. Cryptosporidium spp. and EPEC had the highest pathogenicity indices of 7.9 and 7.6, respectively, followed by rotavirus with an index of 4.3. It was also of interest to note that, although both typical $(18 / 25)$ and atypical $(7 / 25)$ EPEC (defined as the presence or absence of the $b f p$ gene encoding the EAF plasmid; Nataro \& Kaper, 1998) were seen among the cases with diarrhoea, asymptomatic infections were associated only with atypical EPEC (2/2). However, another study found atypical EPEC to be more common than typical EPEC among cases of sporadic diarrhoea in Indian children (Wani et al., 2006). This indicates that the virulence determinants of EPEC strains have to be re-examined, especially for possible geographical variations.

When results from our study were compared with those from a previous study conducted two decades earlier (1983-1985) in the same hospital (Mathew et al., 1991), there were significant changes among the pathogens most

Table 3. Pathogenicity indices for aetiological agents of diarrhoea

\begin{tabular}{|llc|}
\hline Organism & \multicolumn{1}{c|}{ Test } & $\begin{array}{c}\text { Pathogenicity } \\
\text { index }^{*}\end{array}$ \\
\hline Rotavirus & ELISA and PCR & 4.3 \\
Norovirus & PCR & 2.3 \\
Campylobacter spp. & PCR & 1.8 \\
EPEC & Culture and PCR & 7.6 \\
EIEC & PCR & 1.7 \\
DAEC and ETEC & PCR & 1.2 \\
EHEC & PCR & 0.9 \\
Cryptosporidium spp. & Microscopy and PCR & 7.9 \\
\hline
\end{tabular}

${ }^{\star}$ Pathogenicity index for specific pathogen $=\%$ in cases $/ \%$ in controls. commonly associated with diarrhoea. In the previous study, which used conventional culture methods for bacterial pathogens, Shigella spp. (19.7\%), Campylobacter spp. $(15.2 \%)$ and ETEC (14.4\%) were most frequently associated with diarrhoea in children. In the present study, these pathogens together accounted for only $12.0 \%$ of diarrhoeal episodes (19/158). Although rotavirus (17.8\%) was a common cause of diarrhoea in the previous study, our study documented an increase to $43.3 \%$. When pathogenicity indices were compared in the two studies, only rotavirus was also associated with diarrhoea in the previous study, with a pathogenicity index of 14.8. This change in trends and aetiology highlights the need to monitor the prevalence of pathogen-specific infection and disease over time. In addition, the availability of new molecular tools permits easier identification of these pathogens for which cumbersome and technically demanding assays were previously required. However, in spite of the application of six PCR assays, no aetiological agent was identified in $23.4 \%$ of diarrhoeal samples. This gap is comparable to another study in the UK (Amar et al., 2007), where the application of PCR reduced the diarrhoea diagnostic gap from 49 to $25 \%$, and other studies in Denmark where a diarrhoeal pathogen was detected in $54 \%$ of all cases using molecular methods (Olesen et al., 2005). Our study is also similar to other case-control studies where viral aetiology was more common than bacterial or parasitic and where a high prevalence of multiple infections was detected (Olesen et al., 2005; Amar et al., 2007).

One of the limitations of this study was the fact that only a few organisms (rotavirus, EHEC and Cryptosporidium spp.) were included in the comparison of conventional and molecular tests. Further studies with a wider array of tests are required to assess the performance of these newer molecular tests. On the other hand, the application of highly sensitive molecular tests resulted in the detection of a large number of asymptomatic infections among the controls. In the absence of symptoms, it is difficult to determine their significance in the context of hospitalized children. Some of the bacterial pathogens, such as Campylobacter spp., EIEC, ETEC and DAEC, were prevalent among the controls at rates almost as high as among the diarrhoeal cases. However, a high rate of asymptomatic infection has been demonstrated in many previous studies in developing countries, and this study actually showed a greater than $20 \%$ decrease when compared with the previous study in this setting (Mathew et al., 1991).

In conclusion, the molecular methods applied in this study reduced the number of diarrhoeal samples in which no putative enteric pathogens were detected from 59.5 to $23.4 \%$, thereby improving our understanding of the aetiology of infectious diarrhoea in this part of the world. Future larger-scale studies with more assays and community-based studies will permit a clearer understanding of the aetiology of diarrhoea in children in developing countries, 
and will provide data for disease burden estimates and help determine vaccine priorities.

\section{ACKNOWLEDGEMENTS}

The study was supported by the Wellcome Trust Trilateral Initiative on Infectious Diseases no. 063144 and Fogarty International Research Cooperative Agreement, National Institutes of Health (5R03TW2711). We also thank the Health Protection Agency, Centre for Infections, Colindale, UK, for providing reference strains and Roberto Vidal at the Programa de Microbiologia, Institode Ciencias Biomedicas, Facultad de Medicina, Universidad de Chile, for generously giving us the protocol for standardization of the multiplex PCR for diarrhoeagenic E. coli.

\section{REFERENCES}

Amar, C. F., East, C. L., Gray, J., Iturriza-Gomara, M., Maclure, E. A. \& McLauchlin, J. (2007). Detection by PCR of eight groups of enteric pathogens in 4,627 faecal samples: re-examination of the English casecontrol Infectious Intestinal Disease Study (1993-1996). Eur J Clin Microbiol Infect Dis 26, 311-323.

Berkman, D. S., Lescano, A. G., Gilman, R. H., Lopez, S. L. \& Black, M. M. (2002). Effects of stunting, diarrhoeal disease, and parasitic infection during infancy on cognition in late childhood: a follow-up study. Lancet 359, 564-571.

Boom, R., Sol, C. J., Salimans, M. M., Jansen, C. L., Wertheim-van Dillen, P. M. \& van der Noordaa, J. (1990). Rapid and simple method for purification of nucleic acids. J Clin Microbiol 28, 495-503.

Buesa, J., Colomina, J., Raga, J., Villanueva, A. \& Prat, J. (1996). Evaluation of reverse transcription and polymerase chain reaction (RT/PCR) for the detection of rotaviruses: applications of the assay. Res Virol 147, 353-361.

DiStefano, D. J., Kraiouchkine, N., Mallette, L., Maliga, M., Kulnis, G., Keller, P. M., Clark, H. F. \& Shaw, A. R. (2005). Novel rotavirus VP7 typing assay using a one-step reverse transcriptase PCR protocol and product sequencing and utility of the assay for epidemiological studies and strain characterization, including serotype subgroup analysis. J Clin Microbiol 43, 5876-5880.

Green, J., Gallimore, C. I., Norcott, J. P., Lewis, D. \& Brown, D. W. (1995). Broadly reactive reverse transcriptase polymerase chain reaction for the diagnosis of SRSV-associated gastroenteritis. $J$ Med Virol 47, 392-398.

Henriksen, S. A. \& Pohlenz, J. F. (1981). Staining of cryptosporidia by a modified Ziehl-Neelsen technique. Acta Vet Scand 22, 594-596.

Husain, M., Seth, P. \& Broor, S. (1995). Detection of group A rotavirus by reverse transcriptase and polymerase chain reaction in feces from children with acute gastroenteritis. Arch Virol 140, 12251233.

Iturriza Gomara, M., Wong, C., Blome, S., Desselberger, U. \& Gray, J. (2002). Molecular characterization of VP6 genes of human rotavirus isolates: correlation of genogroups with subgroups and evidence of independent segregation. J Virol 76, 6596-6601.

Jones, G., Schultink, W. \& Babille, M. (2006). Child survival in India. Indian J Pediatr 73, 479-487.

Kang, G., Iturriza-Gomara, M., Wheeler, J. G., Crystal, P., Monica, B., Ramani, S., Primrose, B., Moses, P. D., Gallimore, C. I. \& other authors (2004). Quantitation of group A rotavirus by real-time reverse-transcription-polymerase chain reaction: correlation with clinical severity in children in South India. J Med Virol 73, 118-122.

Kosek, M., Bern, C. \& Guerrant, R. L. (2003). The global burden of diarrhoeal disease, as estimated from studies published between 1992 and 2000. Bull World Health Organ 81, 197-204.

Kurugol, Z., Geylani, S., Karaca, Y., Umay, F., Erensoy, S., Vardar, F., Bak, M., Yaprak, I., Ozkinay, F. \& other authors (2003). Rotavirus gastroenteritis among children under five years of age in Izmir, Turkey. Turk J Pediatr 45, 290-294.

Levine, M. M. (2006). Enteric infections and the vaccines to counter them: future directions. Vaccine 24, 3865-3873.

Linton, D., Lawson, A. J., Owen, R. J. \& Stanley, J. (1997). PCR detection, identification to species level, and fingerprinting of Campylobacter jejuni and Campylobacter coli direct from diarrheic samples. J Clin Microbiol 35, 2568-2572.

Mathew, M., Mathan, M. M., Mani, K., George, R., Jebakumar, K., Dharamsi, R., Kirubakaran, C., Pereira, S. \& Mathan, V. I. (1991). The relationship of microbial pathogens to acute infectious diarrhoea of childhood. J Trop Med Hyg 94, 253-260.

Monica, B., Ramani, S., Banerjee, I., Primrose, B., Iturriza-Gomara, M., Gallimore, C. I., Brown, D. W., Fathima, M., Moses, P. D. \& other authors (2007). Human caliciviruses in symptomatic and asymptomatic infections in children in Vellore, South India. J Med Virol 79, 544-551.

Nataro, J. P. \& Kaper, J. B. (1998). Diarrheagenic Escherichia coli. Clin Microbiol Rev 11, 142-201.

Noel, J. S., Fankhauser, R. L., Ando, T., Monroe, S. S. \& Glass, R. I. (1999). Identification of a distinct common strain of "Norwalk-like viruses" having a global distribution. J Infect Dis 179, 1334-1344.

Olesen, B., Neimann, J., Bottiger, B., Ethelberg, S., Schiellerup, P., Jensen, C., Helms, M., Scheutz, F., Olsen, K. E. \& other authors (2005). Etiology of diarrhea in young children in Denmark: a casecontrol study. J Clin Microbiol 43, 3636-3641.

O'Ryan, M., Prado, V. \& Pickering, L. K. (2005). A millennium update on pediatric diarrheal illness in the developing world. Semin Pediatr Infect Dis 16, 125-136.

Pang, X. L., Joensuu, J., Hoshino, Y., Kapikian, A. Z. \& Vesikari, T. (1999). Rotaviruses detected by reverse transcription polymerase chain reaction in acute gastroenteritis during a trial of rhesus-human reassortant rotavirus tetravalent vaccine: implications for vaccine efficacy analysis. J Clin Virol 13, 9-16.

Ruuska, T. \& Vesikari, T. (1990). Rotavirus disease in Finnish children: use of numerical scores for clinical severity of diarrhoeal episodes. Scand J Infect Dis 22, 259-267.

Vidal, M., Kruger, E., Duran, C., Lagos, R., Levine, M., Prado, V., Toro, C. \& Vidal, R. (2005). Single multiplex PCR assay to identify simultaneously the six categories of diarrheagenic Escherichia coli associated with enteric infections. J Clin Microbiol 43, 5362-5365.

Wani, S. A., Nabi, A., Fayaz, I., Ahmad, I., Nishikawa, Y., Qureshi, K., Khan, M. A. \& Chowdhary, J. (2006). Investigation of diarrhoeic faecal samples for enterotoxigenic, Shiga toxin-producing and typical or atypical enteropathogenic Escherichia coli in Kashmir, India. FEMS Microbiol Lett 261, 238-244.

Xiao, L., Escalante, L., Yang, C., Sulaiman, I., Escalante, A. A., Montali, R. J., Fayer, R. \& Lal, A. A. (1999). Phylogenetic analysis of Cryptosporidium parasites based on the small-subunit rRNA gene locus. Appl Environ Microbiol 65, 1578-1583. 